\title{
Future Outlook of Nanotechnology in Agriculture
}

\author{
Chilkuri Sumeeth Reddy ${ }^{*}$, Prashanth Kumar Rai, T. Krishna Sai and Ch. Sai Kiran
}

\author{
Dept of Genetics and Plant Breeding, Sam Higginbottom University of Agriculture \\ Technology and Sciences, Prayagraj, India
}

*Corresponding author

\section{A B S T R A C T}

\section{Keywords}

Atomic \&

Molecular scale,

Toxicity, Soil

interaction, DNA

damage, eco-

toxicological

implications

Article Info

Accepted:

12 November 2020

Available Online:

10 December 2020
Nanotechnology diversified Agriculture, as this technology concepts can be used from nanobiotechnology applications which will influence at the level of molecular markers to DNA sequencing, food processing, storage, to transport, as nanoparticles carry unique properties and miniature dimension, scientists will have to interfere with the matter at atomic and molecular scale as they fall in a transition zone between individual molecules and bulk materials. Seeds can be coated by Silica, Silver, Gold, Chitosan, within a range of $10-100 \mathrm{~nm}$, to provide crop protection by acting as protectants or as a carrier for pesticides or other actives, that too a controlled release of Agrochemicals and target specific action, low water soluble insecticides require organic solvents to help solubilise the pesticide which is toxic to the ecosystem as an alternative nanoparticles can be used, thus improving solubility and reducing toxicity. To initiate the Nanoparticles experimental protocol Scientists have synthesised $\mathrm{ZNO}, \mathrm{CuO}, \mathrm{Ag}$ nanoparticles and applied to Fodder Sorghum and Cowpea at TNAU Coimbatore to assess the seed germination and seed health, all of them improved the germination percentage as compared to control. Effect of Nanoparticles was concentration dependant hence at higher doses of $\mathrm{TiO} 2$ in sorghum enhanced the germination, $\mathrm{ZnO}$ and $\mathrm{CuO}$ improved the root/shoot ratio in sorghum. In case of cowpea, $\mathrm{ZnO}$ and $\mathrm{CuO}$ nanoparticles show significant influence on root/shoot ratio. Ag Nanoparticles act as a strong antimicrobial agents, apart from that nanobiosensors can also be used for tracking the plants moisture content, temperature, RH, and also we can have a closer look of the host-parasitic interaction at molecular level Some of the hurdles include, soil interaction, toxicity, eco-toxicological implications, formation of free radicals leading to DNA damage.

\section{Introduction}

Agriculture is the backbone of Indian economy, as more than 600 million people in India are dependent on it for source of income apart from it agriculture is the main source of food for mankind and animals too it also plays a major role in industrial sector also by acting as a main components for lubricants and resins it defines that agriculture plays a pivotal role in modern era both directly and indirectly. Life cycle in agriculture starts with seed and ends with seed thus it is a basic input in agriculture so we must make sure that seed 
quality and protection is arguably an important phenomenon and we must dive into the diversified subject of science to know the best possible ways to make the seed inevitable to perform at its best under these climatic condition's with erratic rains and make resistance for seed borne diseases and pathogens, as they account to $20-40 \%$ losses every year globally along with that more than $90 \%$ of the pesticides are going in vain thus increasing farmer's expenses and also it's a non eco-friendly method as the applied pesticides are polluting environment and soil, well so everyday has become a challenge for scientists and researchers to find new innovative and reliable ways for seed protection by this way we were able to find a possibility in nanotechnology, first of all what is nano? It is derived from Latin word nannus which reflects the meaning for dwarf, a size of 10-100 $\mathrm{nm}$ and nanotechnology is the study of science, engineering and technology conducted at a nanoscale, it all started in 1959 when a noble prize winner physicist Richard Feynman sparkled the debate by saying 'There's plenty of room at the bottom' in this discussion he has thrown light on how we can manipulate atoms and molecules making a boon for human mankind, and the term nanotechnology was coined by Norio Taniguchi in 1974, later the growth of nanotechnology has seen good rise as in late 1900s and early many nanotechnology initiatives have started leading to a worldwide breakthrough of nanotechnology activities took place as office of science and technology (OSTP) established an Interagency working group on nanotechnology (IWGN), for exploring the advantages of nanotechnology, as it is a multidisciplinary field they have the potential to manipulate matter at molecular level that too in a precise manner, they will act as protectants as well as carriers for nanopesticides, nanofertilizers, and will also function as nanobiosensors for tracking $\mathrm{pH}$ in soil, moisture content, we can have a deeper insight of host parasitic interaction at the molecular level, also the metal oxide based nanosensors will act as protectants from diseases caused by bacteria, will act as a reclamation factor for salt- effected soils, nanotechnology can also be used in food processing, it's importance in nanobiotechnology is also more as they'll act as molecular machine systems, to keep up the food security for this growing population and decreasing cultivable land along with them the major growing global concern is that to meet the requirements of the world appetite with good nutritional values and in a nontoxic manner, adaption to nanotechnology leaves us no choice, we have to find the ways to get over some of the drawbacks of using nanoparticles such as nanoparticle toxicity, bioaccumulation.

\section{Nanoparticles acting as seed protectants}

As seed is the basic input in agriculture it is very important to protect the seed with certain fungicides and insecticides, they're being used since a long time, as such their application is leading to soil toxicity and environmental pollution due to unregulated use of those harm-full chemicals along with that more than $90 \%$ of the pesticides and fertilizers will get evaporated thus polluting the environment, incurring huge expenditure to the farmers, and also the soil fertility is gradually decreasing, thus by using this technology we can improve the seed performance as nanoparticles that we apply to the seed before sowing as they will act as protectants and carrier of Nano pesticides, and will protect the seed form dangerous pests in a less time consuming manner as further spraying is no needed, and also protecting the beneficial insects thus benefitting the farmer in both the ways so using nanoparticles, the concentration is much more important in the effectiveness of nanoparticles and also on time of application, mainly applying post 
fungal infection is showing better results compared to applying before infection and at the time of inoculation, nanoparticles have the potential to protect the seeds from pests and fungal infection by direct application to seeds or foliage, their importance in seed quality enhancement proved to show good results when synthesised nanoparticles dry dressed on onion seeds, the dose of $1000 \mathrm{mg} / \mathrm{kg}$ excelled the germination upto $75 \%$, aged Rice seeds primed with phytosynthesized AgNPs at a concentration of 5 and $10 \mathrm{ppm}$ promoted germination performance and seedling vigour compared to control, the metal nanoparticles which includes $\mathrm{Cu}, \mathrm{ZnO}, \mathrm{TiO} 2$, are under vigorous exploitation for their antifungal, antibacterial properties and antiviral properties, Ag nanoparticles are very important in the world of nanoparticles for their wide adaptability for various properties which include sensor's, anti cancer, anti microbial, and shown a good result 's against fungi, bacteria, but synthesis of silver nanoparticles has become a question for their ammonia production which causes toxicity, later on by the green synthesis production using kaffir lime leaf extract has found an good alternative which also improved the germination of aged rice seeds.

\section{Nanobiosensors role in agriculture}

In the world of pre-knowing and sensibility with the help of modern technology we are able to predict the status of the biological processes and assess certain factors which are important for the plant, prior any damage or loss in the performance, the environmental pollution has also become a major concern with the uncontrolled pollutants, new innovations must be required to measure and control the environmental pollution, along with them we can track the state of any food material that is under transportation or which is kept under storage for long time, food material or seeds kept under those conditions will have the major chances of losing the freshness, so to keep an eye on their degree of freshness, nanobiosensors have come into force for their unique properties, through which they are able to measure accurately, quick in response, it is also compatible with other technologies which includes lab-on-achip through which we can open the doors to molecular analysis, we can also detect a (Image-MIT) wide range of activities such as the amount of urea, glucose, zeolites are gaining importance as nanobiosensors in agriculture for their unique properties through which they are able to detect and control the release of water, nutrients. MIT engineers came up with a sensor which we can print on the leaf and continuously monitor the plant water available in the plant, this is a boon for farmers and mainly for gardener's, as all know the basics about stomatal operation that they will open the light and close in the darkness, to initiate the protocol MIT engineers have designed ink made of carbonnanotubes which can be printed across the stomatal pore to create a electronic current, that can conduct electricity, dissolved in an organic compound sodium dodecyl sulphate which does not cause any harm to the stomata, engineers were able to observe precisely about the stomatal behaviour when exposed to water stress conditions and normal conditions of water available, within 2 days, under normal conditions stomata will open 7 mins after light exposure and will take 53 mins to close when darkness falls, under water stress conditions it'll take upto 25 mins to open and 45 mins to close, when the stomatal pore is in closed condition the circuit is intact which can be measured by multimeter, and when the pore is opened the circuit is broken and the current will flow, through this we can precisely monitor the time of stomatal opening and closing, MIT engineering's designed a complex with nanobiosensors implanted in them with the help of microfluid chip which is implanted on 
the abaxial surface of the leaf with the help of plastic holders which holds two contact pads with conductive straps on them, when the stoma opens the current flow will break and thus we are able to monitor stomatal behaviour responding to different environmental conditions.
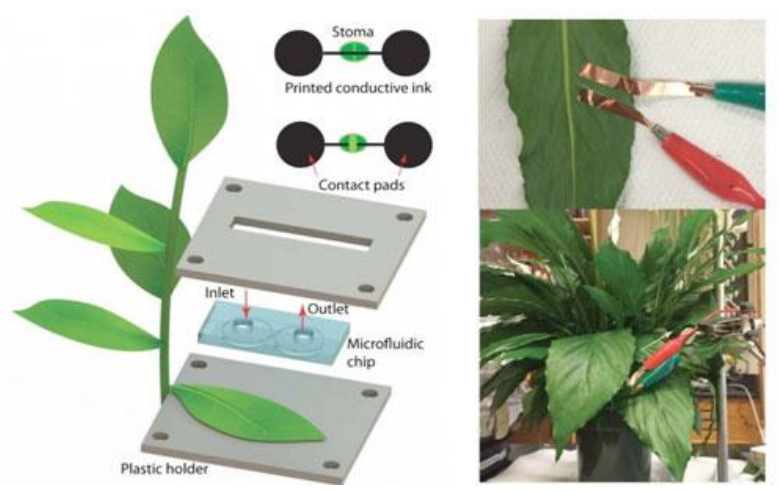

\section{Nanoparticles in seed storage}

Nowadays storage has become an important criteria rather than production, because, if the storage conditions are not good the seeds will get deteriorated and will incur huge losses to the farmer's, so it's equally important to the farmer's to store their seeds until the market prices will rise and bring good returns to them, due to lack of good storage conditions he's forced to sell to the middle man or in a less market value time, thus he's not meeting expenditures, this shows the importance of good storage facilities that must be provided to the farmer so that he solely can get benefitted from the market fluctuations, not only providing good storage structures we must also come up with some extra measures to prevent the seeds from deteriorating, there is bridge between seed deterioration and the release of volatile compounds (Hailstone and Smith 1989), during the storage, seed will emit volatile compounds by lipid peroxidation through several processes which will lead to seed deterioration, by using nanosensors such volatile compounds can be detected and the deteriorating seeds can be separated, an experiment has been conducted in UAS Dharwad to test the effectiveness of nanoparticles on rice hybrid variety $\mathrm{KRH}-4$ with regarding to storage, of all the treatments of nanoparticles they have given, $\mathrm{ZnO} 1000$ $\mathrm{Mg} / \mathrm{kg}$ has shown better results by lowering the rate of seed ageing, and boosted the germination percentage $(99.75 \%)$ initial and $(79.75 \%)$ after storing the seeds for 12 months (puttapanarva and deshpande). Thus $\mathrm{ZnO} 1000 \mathrm{Mg} / \mathrm{kg}$ can be used in multiple ways as it increases vigour, germination and storability.

\section{Nanotechnology in food industries}

Food packaging and labelling is the most important aspect in agriculture as packaging helps to maintain the shelf life of the food products during storage for long period and even under transportation conditions because in this modern era of imports and exports it is equally important to maintain the quality of the food during those conditions, as food is the basic source of feed for humans and animals, as they are the major carriers of energy in the form of protein, carbohydrates, fats, oils, as packaging food material for storage or transportation has become a major concern because of major demands from other countries so we have the necessity to find adaptable methods for safe food packaging without damage to the food quality, and both the developed and developing nations are working on this factor as the spoilage of food, and consuming that food is causing major health issues, as nanotechnology have the ability to interact with matter at molecular level it can be used in sensing food contamination, remove pathogens from food, adding nutritional factor's, they can also be used in food processing, antimicrobial packaging is an important approach as most of nanoparticles especially silver nanoparticles have the antimicrobial 
properties which will interact with wide range of biological processes of bacteria and hinder them with the ions present in it, carbon nanotubes and nanoscale, $\mathrm{TiO}_{2}$ particles will act as good barriers of UV light in plastic packaging. As being found a better option we can switch to nanotechnology as a more effective method and precise in the packaging process. Nanobarcodes are getting involved in labelling and tracking the food products.

In conclusion the emerging room of advanced technologies, nanotechnology will be the most favourite one to open the door in the future, new innovations are growing back their infancy to create even more precise look at the mechanisms that we can't see with our naked eyes, satisfying the mentioned criteria nanotechnology will be use-full In many applied sciences such as agriculture, food industry, medical sciences, because nanotechnology has the ability to combine many science disciplines so it's called as a multidisciplinary concept, nanoparticles, nanobiosensors, nanobiotechnology, will become a breakthrough and will replace the existing technology, so hoping for the better future we must pay some attention for this technology spending time and funds to make this technology reliable and pocket friendly, mainly in india the major concern is that the funds for research is always limited, that's the main reason we are always depending on other countries technologies. Hoping for a better future of technologies and new innovations.

\section{References}

Aniruddha Maity, Natarajan, M Pastor, D Vijay, CK Gupta, and Wasnik, VK (2017). Nanoparticles influence seed germination traits and seed pathogen infection rate in forage sorghum(Sorghum bicolor)and cowpea (Vigna unguiculata), Indian journal of experimental Biology, Vol,56,pp363,367

Anne Trafton, MIT, (2017), Nanosensors applied to plant leaves warn of water shortage, journal Lab on a Chip, ("Persistent drought monitoring using a microfluidic-printed electro-mechanical sensor of stomata in planta").

Chellarama, C., G. Murugaboopathib, A.A. Johna, R. Sivakumarc, S. Ganesand, S. Krithikae and Priyae, G. (2014) 'Significance of Nanotechnology in Food Industry' 2013 4th International Conference on Agriculture and Animal Science (CAAS 2013) 2013 3rd International Conference on Asia Agriculture and Animal (ICAAA 2013) APCBEE Procedia 8 (2014) 109 - 110

Dragomir Mirela. Metallic Nanoparticles University Of Nova Gorica Doctoral Study, Programme Physics

Elizabeth A. Worrall, Aflaq Hamid, Karishma T. Mody, Neena Mitter and Hanu R. Pappu (2018), www.mpdi.com/journal/ Agronomy, Nanotechnology for plant disease management, Pp. 1,3,8,17

Anandaraj, K. and N. Natarajan (2017). Effect of Nanoparticles for Seed Quality Enhancement in Onion [Allium cepa (Linn) cv. CO (On)] 5 International Journal of Current Microbiology and Applied Sciences, Volume 6 Number 11 (2017) pp. 3714

Karimi N,Minaei S, Almassi M. and Shahverdi A.R. (2011). Application of silver nano-particles for protection of seeds in different soils, African journal of Agricultural Research, vol.7(12), pp. 1863,1864

Katherine Alvarado1, Mónica Bolaños1, Carmen Camacho1, Elías Quesada1, José Vega-Baudrit1,2 (2019) 'Nanobiotechnology in Agricultural Sector: Overview and Novel Applications' Journal of Biomaterials and Nanobiotechnology, 2019, 121,127 
Louise Colville1,*, Emma L. Bradley2, Antony S. Lloyd2, Hugh W. Pritchard1, Laurence Castle2 and Ilse Kranner1,3 (2012), Volatile fingerprints of seeds of four species indicate the involvement of alcoholic fermentation, lipid peroxidation, and Maillard reactions in seed deterioration during ageing and desiccation stress, Journal of Experimental Botany 63(18):6519-30

Madhukeshwara B Puttappanavara and V. K. Deshpande(2019) 'Effect of nanoparticles on storability of KRH-4 hybrid rice seeds' Bulletin of Environment, Pharmacology and Life Sciences Vol 8 [5] April 2019: 99-100

Manju Kumari Choudhary, Manvendra Singh and Vinod Saharan, (2015), Application of Nanobiosensors in Agriculture, Popular Kheti, Volume -3, Issue-1 (January-March), 2015,132

Manoj Kaushal, Suhas P. Wani, (2017), Nanosensors: Frontiers in Precision Agriculture, Nanotechnology pp 279, ISBN: 978-981-10-4572-1

Manzer H. Siddique, Mohamed H. AlWhaibi, Mohammad Firoz and Mutahhar Y, Al- Khaishany (2015), Role of Nanoparticles in Plants, Springer international publishing Switzerland
Michael Berger (2014), Nanotechnology in agriculture, Copyright (C) Nanowerk

Michele Greque de Morais1*, Vilásia Guimarães Martins1, Daniela Steffens2, Patricia Pranke23, and Jorge Alberto Vieira da Costa1 (2014) 'Biological Applications of Nanobiotechnology' Journal of Nanoscience and Nanotechnology Vol. 14, 1012-1013

Vineeta Rai, Sefali Acharya, Nrisingha Dey. (2012) 'Implications of Nanobiosensors in Agriculture' Journal of Biomaterials and Nanobiotechnology, 2012, 3, 315316

Vivek K. Bajpai, MadhuKamle, ShrutiShukla, Dipendra Kumar Mahato, Pranjal Chandra, Seung KyuHwang, Pradeep Kumar, Yun SukHuh and YoungKyuHan (2018), Prospects of using nanotechnology for food preservation, safety, and security Journal of Food and Drug Analysis, Volume 26, Issue 4 Wuttipong Mahakham,Ajit K. Sarmah, Santi Maensiri and Piyada Theerakulpisut (2017) Nanopriming technology for enhancing germination and starch metabolism of aged rice seeds using phytosynthesized silver nanoparticles, Article number: 8263

\section{How to cite this article:}

Chilkuri Sumeeth Reddy, Prashanth Kumar Rai, T. Krishna Sai and Sai Kiran, Ch. 2020. Future Outlook of Nanotechnology in Agriculture. Int.J.Curr.Microbiol.App.Sci. 9(12): 14041409. doi: https://doi.org/10.20546/ijcmas.2020.912.169 